La Revue

des Droits

de l'Homme

\section{La Revue des droits de l'homme}

Revue du Centre de recherches et d'études sur les droits fondamentaux

$1 \mid 2012$

Revue des droits de l'homme $-\mathrm{N}^{\circ} 1$

\title{
Chapitre 1. Le droit au service public, reflet des obligations pesant sur les personnes publiques
}

\section{Virginie Donier}

\section{OpenEdition}

\section{Journals}

Édition électronique

URL : http://journals.openedition.org/revdh/151

DOI : 10.4000/revdh.151

ISSN : 2264-179X

\section{Éditeur}

Centre de recherches et d'études sur les droits fondamentaux

\section{Édition imprimée}

Date de publication : 1 juin 2012

Pagination : 393-408

Référence électronique

Virginie Donier, " Chapitre 1. Le droit au service public, reflet des obligations pesant sur les personnes publiques », La Revue des droits de l'homme [En ligne], 1 | 2012, mis en ligne le 30 juin 2012, consulté le 08 juillet 2020. URL : http://journals.openedition.org/revdh/151 ; DOI : https://doi.org/10.4000/revdh. 151 


\section{CHAPITRE 1}

\section{LE DROIT AU SERVICE PUBLIC, REFLET DES \\ OBLIGATIONS PESANT SUR LES PERSONNES PUBLIQUES,}

\section{VIRGINIE DONIER}

Le rôle assigné aux services publics dans la mise en œuvre des principes républicains est fréquemment mis en exergue par le discours politique, mais aussi par la doctrine. Michel Borgetto et Robert Lafore considèrent ainsi que « le service public est le produit du renversement de la conception du droit intervenu à la fin du XIXème siècle, lequel a voulu substituer au droit révolutionnaire d'essence jusnaturaliste, individualiste et contractualiste une nouvelle construction juridique bâtie sur une théorie sociologique de l'intégration sociale »1088. Le discours doctrinal s'accorde en effet sur la fonction de solidarité du service public dans la mesure où il constitue sans nul doute le principal instrument de lutte contre les inégalités de fait ${ }^{1089}$. Une telle analyse est également confirmée par la jurisprudence administrative, le juge n'hésitant pas à justifier la création d'un service public par la volonté d'assurer à tous, et notamment aux plus modestes n'ayant pas les moyens financiers de recourir au secteur privé, l'accès à des prestations jugées essentielles ${ }^{1090}$.

Le rôle du service public dans la mise en œuvre des droits se vérifie particulièrement s'agissant des droits sociaux qualifiés de droits-créances. Comme le soulignait Jean Rivero, «les droits de créances ne peuvent recevoir satisfaction qu'après la mise en place d'un appareil destiné à répondre aux exigences des particuliers. Le service public est donc, pour la satisfaction de tels pouvoirs, le procédé le plus normal. Tant que le service public n'est pas créé (...) le droit du créancier ne peut s'exercer ${ }^{1091}{ }^{1}$. Services publics et droits-créances entretiennent des liens étroits; dès lors, on peut s'étonner qu'aucun texte de droit interne ne consacre expressément le droit aux services publics. Certes, il existe bien des textes qui affirment, dans des domaines particuliers, le droit d'accès à un service public, à l'instar de l'alinéa 13 du Préambule de la Constitution de 1946. Mais aucune disposition constitutionnelle ne consacre le

\footnotetext{
1088 Michel BORGETTO et Robert LAFORE, La République sociale, contribution à l'étude de la question démocratique en France, PUF, collection La politique éclatée, 2000, p. 306 et s.

1089 Laurent THEVENOT, "Les justifications du service public peuvent-elles contenir le marché ? " in Services publics et droits fondamentaux dans la construction européenne, Antoine LYON-CAEN et Véronique CHAMPEIL-DESPLATS (dir.) Dalloz, collection Thèmes et commentaires, 2001, p. 127. Gilles J. GUGLIELMI, Geneviève KOUBI et Gilles DUMONT, Droit du service public, Montchrestien, Domat de droit public, 2007, p. 661.

1090 CE, 20 novembre 1964, Ville de Nanterre, AJDA, 1964, p. 705.

1091 Jean RIVERO, Libertés publiques, t. 1, PUF, 1991, p. 121.
} 
droit d'accès aux services publics alors même que le Préambule de 1946 reconnaît et garantit des droits-créances. Seule la Charte des droits fondamentaux de l'Union européenne fait état d'un droit d'accès à certaines activités de service public : l'article 34 énonce ainsi que "l'Union reconnaît et respecte le droit d'accès aux prestations de sécurité sociale et aux services sociaux (...)»; quant à l'article 36, il affirme que "l'Union reconnaît et respecte l'accès aux services d'intérêt économique général tel qu'il est prévu par les législations et les pratiques nationales, conformément au traité instituant la Communauté européenne, afin de promouvoir la cohésion sociale et territoriale de l'Union ". Ces deux articles constituent les seuls textes susceptibles de servir de fondement à la reconnaissance d'un droit aux services publics, sans pour autant que l'on puisse déduire des formulations retenues une quelconque obligation à la charge des États membres.

Dès lors, faut-il admettre, comme certains auteurs, que les droits sociaux proclamés en France ne le sont à l'encontre d'aucun débiteur clairement identifié$^{1092}$ ? Si l'on se reporte à l'alinéa 11 du Préambule de la Constitution de 1946 qui fait peser sur la Nation la protection de la santé et la sécurité matérielle, on ne peut que souscrire à cette analyse. Certaines dispositions sont en revanche plus précises et désignent sans ambigüité un débiteur, à l'image de l'alinéa 13 selon lequel « l'organisation de l'enseignement public et laïque à tous les degrés est un devoir de l'État». L'emploi du terme "Nation» dans certains alinéas du Préambule témoigne sans doute de la volonté des rédacteurs du texte de laisser une marge de manœuvre substantielle aux pouvoirs publics pour décider des conditions de mise en ouvre des droits considérés. Mais sans instrument d'activation, ces dispositions, et les droits afférents, peuvent rester lettre morte. Au-delà de l'affirmation du lien entre la création d'un service public et la concrétisation d'un droit, il importe donc d'analyser les conditions de mise en œuvre de ce lien et particulièrement l'intensité de la contrainte qui caractérise cette relation. De cette analyse découlera ainsi la réponse à la question de savoir si les personnes publiques sont réellement les débiteurs des droits sociaux, ou pour le moins, les principaux débiteurs. En d'autres termes, s'interroger sur l'identification des débiteurs des droits sociaux ne suppose pas uniquement de mettre en exergue le rôle dévolu aux services publics dans la réalisation de ces droits, il convient en outre de s'interroger sur l'étendue des obligations pesant sur les personnes publiques afin de déterminer si le droit au service public, c'est-à-dire le droit à la concrétisation des droits sociaux, leur est opposable.

D'après Michel Borgetto, l'opposabilité d'un droit implique d'une part, la désignation "d'une autorité responsable de sa mise en œuvre", et d'autre part, "que le citoyen puisse, si nécessaire, revendiquer son droit devant une instance 
compétente » ${ }^{1093}$. Un droit opposable semble donc être également un droit justiciable puisque le citoyen doit pouvoir disposer d'une procédure juridictionnelle permettant de garantir la mise en œuvre de ce droit. Mais la justiciabilité n'est pas nécessairement synonyme d'effectivité, et l'analyse des garanties accordées au droit au service public le démontrera. Ainsi, s'interroger sur l'intensité de la contrainte pesant sur les personnes publiques consiste à déterminer si le droit au service public est opposable, justiciable et effectif. Et pour tenter d'apporter des éléments de réponse, il faudra, dans un premier temps, se demander si la création d'un service public est une obligation à la charge des personnes publiques. Existe-t-il une contrainte juridique pesant sur les personnes publiques en la matière (I) ? Par ailleurs, une seconde question méritera d'être soulevée : la créance pesant sur les personnes publiques est-elle éteinte dès lors que le service public est créé, existe-t-il un droit d'accès aux services publics existants (II)?

\section{L'étendue des devoirs pesant sur les personnes publiques quant à la création des services publics}

Si le droit à la création de services publics est souvent analysé comme un devoir social pesant sur les personnes publiques, il s'agit essentiellement de contraintes a-juridiques. La corrélation entre l'existence de services publics et la concrétisation d'un droit est fréquemment affirmée tant dans le discours doctrinal que dans la jurisprudence (A), mais cela n'engendre pas nécessairement la reconnaissance d'une créance à la charge des personnes publiques (B).

\section{A. Les justifications apportées à l'existence des services publics : l'affirmation d'un devoir social}

Le service public est traditionnellement analysé comme un vecteur de solidarité, et ce quel que soit l'objet de son activité. Cela se confirme particulièrement dans le cadre des services sociaux dont l'objectif est d'assurer une redistribution des revenus. La Commission européenne considère ainsi que les services sociaux d'intérêt général, qui ont pour objet de "répondre aux besoins vitaux de l'Homme", sont "généralement fondés sur le principe de solidarité et dépendent fortement des financements publics, de manière à garantir l'égalité d'accès, indépendamment des richesses ou des revenus " 1094 . Cet objectif de solidarité justifie par ailleurs que de telles activités puissent bénéficier de dérogations aux règles du Traité, et notamment aux règles de la

1093 Michel BORGETTO, "L'accès aux droits sociaux : quelle effectivité ? " in Droit et pauvreté, Séminaire ONPES, DREES-MIiRe, 2008, p. 105 et s.

1094 Communication de la Commission européenne du 20 novembre 2007, "Les services d'intérêt général, y compris les services sociaux d’intérêt général : un nouvel engagement européen ", $\operatorname{COM}(2007) 725$ 
concurrence ${ }^{1095}$, même si ces dérogations ne sont ni systématiques ni généralisées. C'est en effet le degré de solidarité que comporte une activité sociale qui détermine l'applicabilité ou la non-applicabilité des règles du droit de la concurrence ${ }^{1096}$. Cela tend à démontrer que l'existence de services publics à vocation sociale se justifie par la volonté de garantir la mise en œuvre du principe de solidarité, et c'est précisément cet objectif qui justifie également le régime juridique qui leur est applicable en droit communautaire. Le service public est donc présenté comme un outil en faveur de la lutte contre les inégalités de fait.

La jurisprudence administrative confirme cette analyse. Dans une décision rendue le 20 novembre 1964, Ville de Nanterre, le Conseil d'État a considéré que la création d'un service municipal de soins dentaires était justifiée par le souci d'assurer à tous, l'accès à un service essentiel, indépendamment du niveau de ressources des usagers ${ }^{1097}$. De la même manière, dans une décision plus récente, le Conseil d'État a estimé qu'un département pouvait légalement créer un service de téléalarme au profit des personnes âgées afin de faciliter leur maintien à domicile. Cette intervention ne porte pas atteinte au principe de la liberté du commerce et de l'industrie dans la mesure où elle est motivée par le principe de solidarité, c'est-à-dire par la volonté de garantir à toutes les personnes âgées, et notamment aux plus modestes, le bénéfice d'une prestation indispensable à leur maintien à domicile ${ }^{1098}$. Les lois du marché peuvent ainsi céder face aux impératifs de solidarité. Cela met alors en lumière les carences du secteur privé qui n'est pas en mesure de proposer une offre de service accessible à tous; or, il appartient aux personnes publiques de combler ces carences.

Mais la justification des interventions des personnes publiques en matière de création de services publics n'est pas circonscrite au seul secteur social ; le rôle civique que revêt le service public contribue en effet à légitimer les interventions des personnes publiques dans d'autres secteurs. Ce rôle tient à ce que le service public est « un instrument essentiel pour garantir aux citoyens un mieux-être économique et social ${ }^{1099}$, mais il est également présenté comme une composante de la citoyenneté, et notamment de la citoyenneté européenne. C'est ce qui ressort du libre blanc de la Commission européenne du 12 mai 2004 dans lequel il est affirmé que l'accès aux services d’intérêt général " est nécessaire pour permettre aux citoyens de l'Union de jouir pleinement de leurs droits

1095 Patrick THIEFFRY, « Une année en demi-teinte pour les services sociaux d'intérêt général », AJDA, 4 février 2008, p. 176.

1096 Sean VAN RAEPENBUSCH, "Les services sociaux en droit communautaire ou la recherche d'un juste équilibre entre l'économique et le social ", in Les services d'intérêt économique général de l'Union européenne, Jean-Victor LOUIS et Stéphane RODRIGUES (dir.), Bruylant, 2006, p. 69. 1097 Arrêt précité.

1098 CE, 3 mars 2010, Département de la Corrèze, RDSS 2010, n², p 341, note Geneviève KOUBI et Gilles J. GUGLIELMI.

1099 Gilles J. GUGLIELMI, Geneviève KOUBI et Gilles DUMONT, Droit du service public, précit. p. 661. 
fondamentaux ${ }^{1100}$. Cette corrélation autorise alors à penser que l'accès aux services publics est un préalable indispensable à l'exercice de la citoyenneté, et à l'exercice des droits fondamentaux. Dès lors, l'existence des services publics peut trouver sa légitimité dans la volonté de garantir pleinement l'exercice de la citoyenneté, et c'est sans doute ce qui justifie l'article 36 de la charte des droits fondamentaux de l'Union européenne qui se réfère expressément à l'accès aux services d'intérêt économique général; cette disposition ne vise pas nécessairement à faire de l'accès à ces activités un droit fondamental, mais elle a sa place dans la charte dans la mesure où les services d'intérêt général constituent « le socle d'un certain nombre de droits fondamentaux » ${ }^{1101}$.

Une telle analyse se retrouve par ailleurs dans le discours politique français comme en témoigne la proposition de résolution relative au rôle des services publics présentée par plusieurs sénateurs français le 28 mai 2009. Cette proposition vise à affirmer que le "service public est un facteur de cohésion sociale. Il fait une large place à la redistribution sociale et territoriale (...). Le service public n'est donc pas simplement un prestataire de biens et de services mais aussi un producteur de lien social et de citoyenneté ${ }^{1102}$. Cette vision du service public avait déjà été mise en exergue lors des débats parlementaires afférents à la loi "exclusions" du 29 juillet $1998^{1103}$, la création de services publics étant présentée comme un instrument indispensable à la réalisation des droits fondamentaux. Le lien ainsi établi entre services publics et citoyenneté se retrouve également dans la jurisprudence administrative, et plus spécifiquement, dans la jurisprudence relative à la tarification sociale des services publics. Dans ses conclusions rendues à propos des arrêts Commune de Gennevilliers et Commune de Nanterre du 29 décembre 1997, Jacques-Henri Stahl estimait qu'«affirmer qu'est d'intérêt général tout ce qui contribue à affermir le lien social n'est pas déraisonnable "1104. Et cette analyse est appliquée, dans les deux cas d'espèce, à un service public qui n'a pas directement une vocation sociale car il s'agit d'un service culturel, ce qui tend à conforter le rôle assigné aux différents services publics dans la mise en œuvre de la citoyenneté.

La place dévolue aux services publics dans le discours politique, mais aussi dans la doctrine et la jurisprudence, contribue alors à mettre à la charge des personnes publiques, et notamment de l'État, un devoir qui trouve sa source dans la nécessité de garantir les conditions nécessaires à l'exercice de la citoyenneté. C'est donc là que le service public puise les justifications de son existence : dans la mesure où le secteur privé ne peut, à lui seul, assurer l'accès de tous aux droits fondamentaux, l'intervention des pouvoirs publics paraît non

\footnotetext{
1100 COM (2004) 374 final.

1101 Hélène PAULIAT, "L'accès aux services d'intérêt général et la cohésion sociale et territoriale ", in L'accès aux services d'intérêt économique général, Jacques VANDAMME et Stéphane RODRIGUES (dir.), éditions ASPE Europe, 2003, p. 98.

1102 Proposition de résolution $n^{\circ} 446$.

${ }_{1103}$ Loi n $98-657$, JO 31 juillet 1998, p. 11679.

1104 RFDA, 1998, p. 539.
} 
seulement justifiée, mais également indispensable. Se dessine ainsi les contours d'un devoir pesant sur les personnes publiques et en premier lieu sur l'État. La question est alors de savoir si ce devoir social, ou cette contrainte a-juridique, trouve sa concrétisation en droit: le devoir social qui semble être à l'origine de l'existence de services publics se traduit-il par la consécration d'un droit opposable?

\section{B. L’intensité de la contrainte juridique : une opposabilité variable}

La question de savoir si les personnes publiques sont dans l'obligation de créer des activités de service public suppose de rechercher l'existence de dispositions textuelles ou de principes juridiques dictant au législateur, ou aux collectivités territoriales, une telle obligation. D'après certaines analyses doctrinales, existeraient des services publics dont l'existence est imposée, ou tout du moins découle, de dispositions constitutionnelles et sont ainsi placés à l'abri du législateur ${ }^{1105}$. Cela ne signifie pas pour autant que de telles activités constituent, dans tous les cas, des monopoles étatiques, certains services publics dont l'existence découle de dispositions constitutionnelles peuvent en effet admettre, aux côtés du secteur public, un secteur privé1106. Tel est le cas de l'éducation ou de la santé.

Mais il n'en demeure pas moins qu'une partie de la doctrine considère que de telles activités, qui semblent être impliquées par une disposition constitutionnelle, s'imposent aux personnes publiques et supposent l'existence d'un service public; s'agissant du service public de l'éducation, cela est affirmé par l'alinéa 13 du Préambule de 1946. Cependant, le manque de précision de certaines dispositions ne permet pas toujours d'en déduire explicitement l'obligation de créer un service public. A la lecture de l'alinéa 11 du Préambule de 1946, on ne peut en effet conclure à l'existence d'un service public constitutionnel de la protection sociale. Si cette disposition reconnaît le droit à la protection sociale, elle n'exige pas que ce droit soit mis en ouvre par le biais d'un service public ; il appartient certes, à la Nation de garantir ce droit, mais compte tenu de l'imprécision de cette disposition, on ne peut conclure, de prime abord, à l'obligation de créer un service public. Les moyens à mettre en œuvre pour garantir les droits énoncés par l'alinéa 11 ne sont pas clairement exposés, ils sont laissés à la libre appréciation du législateur. Or, cette indétermination n'est pas sans conséquences sur l'opposabilité de cette disposition, le manque de

1105 Louis FAVOREU, "Service public et Constitution", AJDA, 1997, nº spécial, p. 16 ; Pierre ESPUGLAS, Conseil constitutionnel et service public, LGDJ, Bibliothèque constitutionnelle et de science politique, 1994. Pour une analyse contraire, v. Ramu DE BELLESCIZE, Les services publics constitutionnels, LGDJ, Bibliothèque de droit public, 2005.

${ }_{1106}$ Ces services publics entrent alors dans ce que certains auteurs qualifient de services publics constitutionnels de "second rang", par opposition aux services publics constitutionnels de "premier rang" qui correspondent aux fonctions de souveraineté et qui excluent toute intervention du secteur privé. V. en ce sens Pierre ESPUGLAS, précit. p 34 et s. 
précision dans l'énoncé d'un droit étant régulièrement présentée comme un obstacle à son opposabilité et à sa concrétisation ${ }^{1107}$.

Néanmoins, on peut considérer que la dimension universelle de cet alinéa, visant à garantir à "tous" le droit à la sécurité matérielle, impose l'existence d'un secteur public aux côtés du secteur privé qui, par nature, est guidé par des considérations financières. Ainsi, en dépit de l’imprécision de l'alinéa 11, on peut tout de même penser que l'universalité dont cette disposition est empreinte implique la mise en œuvre d'un service public. Pour autant, cette obligation n'est assortie d'aucune sanction, aucun dispositif juridique ne permet, en effet, d'enjoindre au législateur la création de services publics, a fortiori lorsque ces activités découlent de dispositions constitutionnelles dont les implications sont nébuleuses.

Cela tend notamment à mettre en lumière l'importance de l'existence d'une disposition précise, qu'elle soit constitutionnelle ou législative, pour imposer l'obligation de créer un service public. Et sur ce point, la mise en œuvre du service minimum d'accueil conforte cette analyse : dans sa décision rendue le 7 août 2008, le Conseil constitutionnel a considéré que l'institution d'un droit d'accueil au profit des élèves des écoles maternelles et élémentaires publiques pendant le temps scolaire supposait la création d'un service public ${ }^{1108}$. En l'occurrence, la corrélation entre la reconnaissance d'un droit et l'existence d'un service public est affirmée dans la mesure où elle découle d'une disposition législative dont le niveau de précision est suffisant pour créer une obligation à la charge des personnes publiques. Et c'est parce que l'existence d'une telle obligation ne fait pas de doute que le juge administratif n'hésite pas à sanctionner les collectivités territoriales ayant refusé de mettre en place un tel service public, et à faire usage de son pouvoir d'injonction, éventuellement sous astreinte, afin de leur imposer la réalisation de cette obligation ${ }^{1109}$. On remarque dès lors que le juge administratif dispose, par le biais de son pouvoir d'injonction, de la possibilité de garantir la mise en œuvre d'une obligation mise à la charge d'une autorité administrative par la loi. Mais aucune solution similaire n'est valable à l'adresse du législateur dont la carence dans la création du service ne semble pouvoir être sanctionnée, notamment lorsque l'obligation découle de dispositions imprécises.

Deux conditions semblent finalement devoir être réunies pour assurer l'opposabilité d'un droit : il convient d'une part que ce droit soit affirmé en des termes suffisamment précis, et d'autre part, il doit être assorti d'une sanction

1107 Xavier PRETOT, commentaire de l'alinéa 11 du Préambule de la Constitution de 1946, Le Préambule de la Constitution de 1946, Gérard CONAC, Xavier PRETOT, Gérard TEBOUL (dir.) Dalloz, collection Thèmes et commentaires, 2001, p. 261 ; François RANGEON, "Réflexions sur l'effectivité du droit ", in Les usages sociaux du droit, Danièle LOCHAK (dir.), CURAPP, PUF, 1989, p. 126.

1108 CC, n² 2008-569 DC, JO 21 août 2008.

1109 V. par exemple TA Paris, 30 janvier 2009, n 0816692, JCP édition A, 4 mai 2009, p. 39, comm. Jean-Marie PONTIER. 
résidant dans l'existence d'un recours juridictionnel, ce qui tend à mettre en exergue le rôle du juge dans la garantie des droits ${ }^{1110}$.

Mais surtout, l'opposabilité du droit au service public semble variable, ce qui laisse apparaître un paradoxe entre l'affirmation d'un devoir social à la charge de l'État et l'absence d'obligation générale pesant sur les personnes publiques. Les justifications apportées à l'existence des services publics ne se sont pas donc nécessairement traduites en contraintes juridiques : si le discours doctrinal et politique reconnaît largement l'existence d'une créance à la charge des personnes publiques, le droit positif ne dispose pas d'un instrument général d'activation de cette créance. Le service public est certes présenté comme un vecteur de citoyenneté et donc comme le socle d'un certain nombre de droits fondamentaux, mais la traduction de cette analyse en droit positif est soumise à des conditions qui ne sont pas nécessairement satisfaites.

La question que l'on peut se poser à présent, à l'égard de l'existence des services publics, est de savoir si le pouvoir d'appréciation reconnu aux pouvoirs publics pour mettre en ouvre de telles activités, en l'absence de dispositions textuelles précises, s'exerce de la même manière tout au long de la vie du service. La faculté de créer un service public se traduit-elle, parallèlement, par la faculté de le supprimer, ou pour le moins, d'en réduire le champ d'action soit matériellement, soit géographiquement? A cet égard, la jurisprudence constitutionnelle relative aux services publics constitutionnels permet d'établir que la marge de manœuvre du législateur s'agissant du maintien de l'activité est plus retreinte. Michel Borgetto le démontre à propos du service public de la protection sociale: selon lui, la notion de service public constitutionnel tend à préserver le droit à la protection sociale en interdisant au législateur de supprimer purement et simplement ce service sans le remplacer par un système au moins équivalent1111. Il apparaît ainsi que l'existence d'une disposition constitutionnelle légitimant l'existence d'un service public peut le mettre à l'abri de toute suppression radicale. Si l'imprécision de certaines dispositions constitutionnelles ne permet pas de déceler l'affirmation d'un droit opposable à la création de l'activité dans la mesure où la carence du législateur ne peut être sanctionnée, ces dispositions peuvent néanmoins devenir opposables dès lors qu'il s'agit de préserver l'existence même du service.

Pour autant, cela ne signifie pas que l'autorité publique n'est pas en mesure de supprimer certaines implantations locales de services publics; le droit au maintien des services, dont certains trouvent leur fondement dans des dispositions constitutionnelles, est en réalité un droit à la prestation. Ce droit se

\footnotetext{
1110 Véronique CHAMPEIL-DESPLATS, « Normativité et effectivité des droits économiques et sociaux dans les débats constituants de 1946 ", in La Déclaration universelle des droits de l'Homme (1948-2008) - Réalité d'un idéal commun?, La documentation française, 2009, p. 406. V. également François RANGEON, " Réflexions sur l'effectivité du droit ", précit.

1111 Michel BORGETTO, « La notion de service public constitutionnel face au droit de la protection sociale " in Le droit administratif entre permanences et convergences, Mélanges en l'honneur de Jean-François LACHAUME, Dalloz, 2007, p. 83.
} 
concilie avec le pouvoir d'appréciation reconnu aux personnes publiques dans la gestion de leurs activités de service public, comme en témoigne la jurisprudence administrative relative à la réforme de la carte judiciaire: si la justice est incontestablement un service constitutionnel, le juge administratif considère que l'autorité chargée de sa mise en œuvre est tout à fait fondée à décider de supprimer certains tribunaux, c'est-à-dire certaines implantations locales de ce service ${ }^{1112}$. Certes, on trouve dans la jurisprudence administrative, des décisions qui imposent le maintien d'un service dès lors qu'il répond à un besoin avéré ; tel est notamment le sens d'une décision rendue par le Conseil d'État à propos d'un service d'ophtalmologie dans un hôpital ${ }^{1113}$. Toutefois, cette solution paraît quelque peu isolée. La mise en perspective de ces deux jurisprudences permet d'ailleurs de démontrer que les services régaliens ne bénéficient pas nécessairement d'une protection plus importante que celle accordée aux services à vocation sociale. Le pouvoir d'organisation reconnu à l'administration sur ses services publics dépasse largement le clivage entre droits civils et politiques d'un côté, et droits économiques, sociaux et culturels de l'autre. Ce pouvoir lui permet en effet, dans les deux cas, de procéder à des aménagements géographiques en supprimant des implantations locales, et ce, même s'il s'agit de services trouvant leur justification dans la Constitution. Dans la mesure où de telles décisions ne conduisent pas à remettre en cause le droit à la prestation, puisque le service n'est pas supprimé dans sa globalité, elles sont autorisées.

S'agissant en revanche des aménagements matériels qui pourraient être apportés aux activités de service public découlant de la Constitution, le juge constitutionnel a construit une jurisprudence qui semble révéler l'existence d'une contrainte juridique à la charge des personnes publiques. Lorsque le législateur aménage le champ matériel d'une prestation existante, le Conseil estime qu'il doit veiller à ce que ces aménagements n'aboutissent pas à remettre en cause des exigences de caractère constitutionnel ${ }^{1114}$. Cela signifie que le législateur se doit de respecter un minimum incompressible, la loi ne peut affecter « la substance même d'un droit (...) en réduisant [la garantie accordée antérieurement] de manière telle que le droit en question se trouve atteint dans son principe " ${ }^{1115}$. Cette jurisprudence est applicable aux services à vocation sociale, ce qui tend à démontrer que l'alinéa 11 du Préambule de 1946 peut mettre des obligations à la charge des personnes publiques. Cet alinéa devient opposable lorsque le législateur modifie le champ des garanties matérielles

1112 V. en ce sens les décisions rendues par le CE le 8 juillet 2009, Commune de Saint-Die-desVosges, $n^{\circ} 314236$ et Commune de Nogent le Rotrou n³19066 ; Mathieu TOUZEIL-DIVINA, "La redistribution des cartes judiciaires ", AJDA,2010, p 398. V. également CE, 19 février 2010, M. Pierre M et autres, $n^{\circ}$ 322407. Dans ces différentes espèces, si le Conseil d'État annule parfois la décision de suppression de tel ou tel tribunal, il n'en demeure pas moins qu'il rappelle l'étendue du pouvoir d'organisation dont disposent les autorités publiques sur ce service public.

1113 CE, 28 février 1990, Décaudin, nº 73788.

${ }^{1114} \mathrm{~V}$. en ce sens la décision $\mathrm{n}^{\circ}$ 97-393DC du 18 décembre 1997, Loi de financement de la sécurité sociale pour 1998, JO 27 décembre 1997, p. 19663.

1115 Michel BORGETTO et Robert LAFORE, La République sociale. Contribution à l'étude de la question démocratique en France, PUF, collection La politique éclaté, 2000, p. 231 et s. 
accordées au droit à la sécurité matérielle, au droit à la protection de la santé, ou au droit d'obtenir des moyens convenables d'existence. Le service public semble ainsi être protégé dans son existence même, mais aussi, dans une certaine mesure, dans son champ matériel. Pour autant, aucune jurisprudence n'est, pour l'heure, venue sanctionner les choix du législateur; la définition de conditions plus restrictives pour bénéficier d'une prestation n'a pas été jugée contraire à la Constitution ${ }^{1116}$. Les potentialités offertes par cette ligne jurisprudentielle, qualifiée de cliquet anti-retour, restent donc virtuelles. Il n'en demeure pas moins qu'elle est susceptible de servir de fondement à l'opposabilité du droit au service public dès lors que l'activité en cause découle d'une disposition constitutionnelle.

En définitive, l'existence des services publics reçoit une protection variable: si la création de services publics relève généralement du pouvoir discrétionnaire des personnes publiques, la vie de ces activités bénéficie d'une protection qui peut être opposée à la personne publique. Mais au-delà des questions ayant trait à l'existence des services publics, il convient également de s'interroger sur la portée du droit d'accès aux services créés.

\section{L'étendue des devoirs pesant sur les personnes publiques quant à l'accès aux services publics existants}

L'analyse ne porte plus, dans cette hypothèse, sur les conditions d'existence des services publics, c'est-à-dire sur les conditions entourant leur création et leur suppression, mais se tourne vers les obligations pesant sur les personnes publiques à l'égard des services déjà mis en place. Cela permettra de démontrer que le droit d'accès au service public est opposable grâce aux lois du service public qui imposent un certain nombre de contraintes aux personnes publiques, contraintes qui peuvent servir de fondement à un recours juridictionnel. Le droit d'accès est donc justiciable (A), mais cela suffit-il pour garantir son effectivité (B)?

\section{A. La justiciabilité du droit d'accès garantie par les lois du service public}

Pas plus qu'il n'existe de droit général à la création et au maintien des services publics, il n'existe un droit d'accès aux services publics reconnu de manière globale. En dépit des affirmations contenues dans les articles 34 et 36 de la charte des droits fondamentaux de l'Union européenne, on ne peut conclure à la reconnaissance d'un droit d'accès. En effet, ces dispositions visent simplement à reconnaître les actions des États en la matière, sans leur dicter de réelles obligations. A l'instar de certains auteurs, il faut en convenir que l'article

1116 V. en ce sens la décision précité du 18 décembre 1997 : le Conseil constitutionnel a considéré que le législateur pouvait soumettre l'octroi d'allocations familiales à une condition de ressources sans remettre en cause des exigences de caractère constitutionnelle. 
36 «n'est pas à ranger parmi les droits subjectifs invocables en justice»; "l'article 36 ne contraint pas les instances communautaires à mettre en œuvre un droit d'accès aux services d'intérêt économique général, il consacre au contraire une abstention: ne pas remettre en cause les législations et les pratiques nationales qui mettent en œuvre le droit d'accès aux services d'intérêt économique général " ${ }^{1117}$. Le contenu du droit d'accès est ainsi déterminé par chaque État membre, réserve faite du service universel puisque cette notion détermine un minimum commun à tous les États et auquel tous les citoyens de l'Union ont droit. Le service universel confère donc des droits aux usagers, tout du moins, dans les domaines dans lesquels il existe car le champ d'application de cette notion demeure sectoriel ${ }^{1118}$. Mais en dehors du service universel, aucune obligation découlant du droit communautaire n'est imposable aux États, les termes de la charte des droits fondamentaux ne souffrent sur ce point d'aucune ambigüité : l'Union européenne se cantonne dans un rôle d'abstention, elle se refuse à endosser un rôle d'impulsion.

Dès lors, puisque le droit communautaire n'est pas la source d'un droit général d'accès aux services publics, il convient de voir dans quelle mesure les règles qui régissent le fonctionnement des services publics en droit interne peuvent être à l'origine d'un droit d'accès. C'est en effet au travers des principes de continuité, d'égalité, et dans une moindre mesure d'adaptabilité que le droit d'accès au service public semble trouver une traduction. Si la doctrine tend à lier la question du droit d'accès à celle de l'interprétation du principe d'égalité ${ }^{1119}$, il convient également de ne pas écarter les deux autres lois du service public en ce qu'elles contribuent à garantir un droit au fonctionnement normal de l'activité, droit qui est opposable.

S'agissant tout d'abord du principe d'égalité, la jurisprudence démontre que ce principe bénéficie d'une attention particulière : dans la décision précitée du 20 novembre 1964, ville de Nanterre, le Conseil d'État affirme que l'accès à un service de soins dentaires ne peut être interdit à un candidat-usager sous prétexte que ses ressources lui permettent de recourir au secteur privé. Certes, la création de services à vocation sociale réservés aux plus démunis est admise par le juge ${ }^{1120}$, mais en dehors de ces activités, le niveau de ressources ne peut

\footnotetext{
1117 Loï GRARD, "Place et signification de la charte des droits fondamentaux de l'Union européenne pour le concept de service d'intérêt économique général ", in L'accès aux services d'intérêt économique général, précit. p 31. Olivier DE SCHUTTER, "Le droit d'accès aux services d'intérêt économique général comme instrument de promotion des droits sociaux ", in L'accès aux services d'intérêt économique général, précit. p. 69.

1118 C'est notamment pour cette raison que, d'après certains auteurs, le service universel n'a d'universel que le nom : voir en ce sens Gilles J. GUGLIELMI, "Un service public universel ? " in Sujets et objets universels du droit, Geneviève KOUBI et Olivier JOUANJAN (dir.), Presses universitaires de Strasbourg, 2007, p. 91.

1119 V. en ce sens le manuel de Jean-François LACHAUME, Hélène PAULIAT et Claudie BOITEAU, Droit des services publics, Armand-Colin, 2004, p. 404 et s : la question de l'accès est présentée comme une composante de l'égalité devant le service public.

${ }^{1120}$ Dans sa décision du 23 juillet 1999, Loi portant création d'une couverture maladie universelle (décision $\mathrm{n}^{\circ}$ 99-416DC, JO 28 juillet 1999), le Conseil constitutionnel a estimé que le législateur pouvait avoir recours à un plafond de ressources pour déterminer les catégories de personnes
} 
constituer un critère d'exclusion. Par ailleurs, la jurisprudence relative à l'utilisation du critère de la nationalité témoigne de l'étendue des garanties accordées aux usagers grâce au principe d'égalité : le juge administratif n'hésite pas à sanctionner les différences de traitement fondées sur ce critère lorsqu'elles sont sans lien avec l'objet du service ${ }^{1121}$. Mais surtout, certaines décisions démontrent que le juge exerce un contrôle vigilant : tel est le cas d'un jugement du Tribunal administratif de Lyon du 17 décembre 1996. Dans cette espèce, un conseil municipal avait décidé de subordonner le versement de certaines aides en direction des personnes âgées à la perception de l'allocation supplémentaire versée par le fonds national de solidarité1122. Aucune condition de nationalité n'était expressément mentionnée, mais pour pouvoir bénéficier de l'allocation supplémentaire, il fallait être de nationalité française ou être ressortissant d'un État ayant signé une convention internationale de réciprocité avec la France. Il existait donc, de manière indirecte et dissimulée, une discrimination fondée sur la nationalité, comme l'a reconnu le juge qui n'a pas manqué de la sanctionner ${ }^{1123}$. Cette jurisprudence démontre qu'il existe une obligation à la charge de la personne gestionnaire du service en vertu de laquelle la construction de catégories d'usagers doit être justifiée par une différence objective de situation ou par une nécessité d'intérêt général en rapport avec l'objet de l'activité ${ }^{1124}$. Un tel droit est non seulement opposable, mais aussi justiciable puisque l'usager est en mesure de saisir le juge administratif par le biais d'un recours pour excès de pouvoir ou par le biais d'un référé afin de faire sanctionner une discrimination, c'est-à-dire une différence de traitement injustifiée.

Outre ce droit à l'égalité, existe également un droit au fonctionnement régulier du service découlant des principes de continuité et d'adaptabilité ; il implique que les usagers puissent bénéficier de la prestation dans les conditions normalement prévues. Un tel droit, qui pèse tant sur la personne publique gestionnaire de l'activité, que sur le délégataire de service public ${ }^{1125}$, permet par exemple de s'opposer aux interruptions injustifiées ${ }^{1126}$; il confère par conséquent un droit à la prestation, dans les conditions définies par les textes. Mais surtout, il importe de souligner qu'un tel droit est opposable et justiciable dans la mesure

pouvant prétendre à la couverture maladie universelle. Dans l'hypothèse des services publics à vocation sociale, la prise en compte des ressources pour exclure de l'accès à l'activité est justifiée. 1121 CE, 30 juin 1989, BAS de Paris Contre Lévy, RFDA 1990, p 578, concl. Daniel LEVIS ; Danièle LOCHAK, "Les discriminations frappant les étrangers sont-elles licites ? ", Droit social 1990, n¹, p. 76.

1122 TA Lyon, 17 décembre 1996, Association comité tous frères, rec. tables p. 693.

${ }_{1123}$ V. également TA Marseille, ordonnance, 21 janvier 2002, M. Sow, AJDA,avril 2002, p. 362 : dans cette espèce, le tribunal a souligné qu'une distinction établie entre les étrangers résidant régulièrement en France et les étrangers en situation régulière pour déterminer les tarifs d'une cantine scolaire faisait naître un doute sérieux quant à sa légalité.

${ }^{1124}$ CE, 10 mai 1974, Denoyez et Chorques, rec. p. 274.

1125 CE, 21 décembre 1906, Syndicat des propriétaires et contribuables du quartier Croix-deSeguey-Trivoli, GAJA.

${ }^{1126}$ CE, 13 février 1987, Toucheboeuf, rec. p. 45 : le Conseil a, dans cette espèce, eu l'occasion de sanctionner la fermeture anticipée d'un établissement d'enseignement afin d'organiser des épreuves d'examen. 
où il peut notamment servir de fondement à l'exercice d'une action en responsabilité ${ }^{1127}$.

Ainsi, c'est par le biais des lois de Rolland que l'usager du service public peut revendiquer un droit d'accès à la prestation, droit qui est justiciable. Pour autant, cela autorise-t-il à conclure à l'effectivité du droit d'accès, la justiciabilité d'un droit est-elle garante de son effectivité ?

\section{B. Un droit d'accès dont l'effectivité demeure perfectible}

En dépit du caractère opposable des règles de fonctionnement du service public, les décisions dans lesquelles le juge consacre l'existence d'une obligation de résultat à la charge d'une personne publique demeurent rares. En d'autres termes, si le juge s'assure que les personnes publiques n'ont pas méconnu les lois de Rolland, cela ne suffit pas pour mettre au crédit des usagers un droit d'accès effectif. Cela découle notamment de l'interprétation qui est traditionnellement retenue du principe d'égalité. Ce principe peut servir de fondement à la mise en œuvre de différences de traitement tarifaires afin de permettre à tous, l'accès à un service public. Favoriser l'accès à ces activités peut constituer une nécessité d'intérêt général et ainsi, justifier l'application d'une tarification sociale ${ }^{1128}$. Mais en aucun cas, le principe d'égalité ne peut donner lieu à la consécration d'un droit à la différence ${ }^{1129}$ : la mise en place d'une tarification sociale, visant à garantir à tous l'accès à certaines activités, relève du seul pouvoir d'appréciation de la personne gestionnaire de l'activité, réserve faite des hypothèses dans lesquelles un texte impose un tarif social1130. Par conséquent, la justiciabilité du droit d'accès n'est pas nécessairement garante de son effectivité puisque la personne publique n'est pas tenue de remédier à tous les obstacles d'ordre matériel susceptibles de compromettre cet accès.

Les décisions dans lesquelles le juge consacre une véritable obligation de résultat à la charge de la personne publique sont rares, mais il en existe tout de même dans le cadre du service public de l'éducation. Le Conseil d'État a en effet eu l'occasion d'engager la responsabilité de l'État en raison de sa carence dans la mise en œuvre des moyens nécessaires au fonctionnement de ce service public ${ }^{1131}$. Par ailleurs, le juge administratif n'a pas hésité à affirmer, dans une décision du 8 avril 2009, l'existence d'une obligation de résultat en faveur des

\footnotetext{
1127 CE, 27 janvier 1988, Ministre de l'Education nationale contre Giraud, rec. p. 39 : le juge a reconnu la faute de l'État qui n'avait pas nommé suffisamment de professeurs pour assurer les heures d'enseignement obligatoires inscrites au programme scolaire.

1128 Sur cette question, voir les arrêts rendus par le CE le 29 décembre 1997, Commune de Gennevilliers et Commune de Nanterre, avec les conclusions de Jacques-Henri STAHL, précit. note Michel BORGETTO, RDP 1998, n³, p. 899. V. également CE, 20 janvier 1989, CCAS de la Rochelle, AJDA, 1989, p. 398, note Xavier PRETOT ; CE, 18 mars 1994, Mme Dejonckerre, LPA 4 novembre 1994, note Victor HAÏM.

1129 CE, 28 mars 1997, Société Baxter, rec. p. 114.

${ }^{1130} \mathrm{~V}$. par exemple, s'agissant du service téléphonique, les obligations figurant à l'article L35-1 du code des postes et des communications téléphoniques.

${ }^{1131}$ CE, 27 janvier 1988, Ministre de l'éducation nationale contre Giraud, précit.
} 
enfants handicapés ${ }^{1132}$. Cette décision met fin aux tergiversations de la jurisprudence antérieure, certaines décisions ayant considéré que l'État était uniquement redevable d'une obligation de moyens, tandis que d'autres n'avaient pas hésité à affirmer que la carence de l'État dans la mise en œuvre du droit à l'éducation était constitutive d'une faute ${ }^{1133}$. Dans l'arrêt du 8 avril 2009, le Conseil d'État affirme sans ambigüité que la scolarisation des enfants handicapés est un devoir pesant sur l'État, ce qui revient à leur reconnaître un droit à la prestation indépendamment des différences de situation que connaissent les enfants handicapés par rapport aux autres enfants. Le juge affirme en effet que "le droit à l'éducation est garanti à chacun, quelles que soient les différences de situation", et il ajoute que "l'obligation scolaire s'appliquant à tous, les difficultés particulières que rencontrent les enfants handicapés ne sauraient avoir pour effet ni de les priver de ce droit, ni de faire obstacle au respect de cette obligation ". Mais l'affirmation d'une obligation de résultat à l'adresse des personnes publiques reste marginale au sein de la jurisprudence: si, dans l'hypothèse du service public de l'éducation nationale, les principes d'égalité et de continuité se révèlent particulièrement contraignants à l'égard du gestionnaire du service, une telle solution demeure isolée. Dès lors, quelles sont les raisons qui expliquent que les lois du service public puissent faire l'objet d'une interprétation aussi contraignante dans le cadre de l'éducation et pas dans le cadre d'autres activités de service public? L'explication réside peut-être dans l'existence d'une disposition textuelle suffisamment claire pour que sa normativité ne soit pas sujette à controverses. En l'occurrence, les dispositions de l'alinéa 13 du Préambule la Constitution de 1946 mettent clairement à la charge de l'État « l'organisation de l'enseignement public et laïc à tous les degrés ». Ce texte a très certainement servi de fondement à l'évolution de la jurisprudence administrative et à l'affirmation d'une obligation de résultat. Cela démontre que le droit d'accès au service public ne s'épuise pas dans les seules lois de Rolland: ces principes fondamentaux permettent effectivement de garantir la justiciabilité du droit au service public mais leur intensité semble pouvoir varier selon les activités. Si l'on met en perspective la jurisprudence relative à la tarification sociale et la jurisprudence relative à l'accès au service public de l'éducation, on observe que la force contraignante de ces principes n'est pas la même. Certes, c'est au travers des principes d'égalité, de continuité et d'adaptabilité que l'accès au service, et notamment au service public de l'éducation, trouve sa concrétisation, mais on ne peut se satisfaire de ces seules dispositions. C'est la conjonction entre ces principes et l'existence d'une norme claire et précise qui a permis au juge

1132 CE, 8 avril 2009, Laruelle, AJDA, 29 juin 2009, p. 1261, concl. Rémi KELLER ; Hervé RIHAL, "La scolarisation des enfants handicapés: une obligation de résultat pour l'État», RDSS 2009, p. 556 ; Philippe RAIMBAULT, «La reconnaissance d'une droit subjectif à la scolarisation des enfants handicapés ", Recueil Dalloz 2009, p. 1508.

1133 V. en sens le jugement du TA de Lyon, 29 septembre 2005, M et Mme K, JCP éd A, 26 décembre 2005, p. 1393, note Samuel DELIANCOURT. Dans cette décision, le tribunal avait considéré que l'État était simplement tenu d'une obligation de moyens. Parallèlement, voir CAA Paris, 11 juillet 2007, Ministre de la santé et des solidarités, AJDA, 2007, p. 2151 : cette décision consacre une obligation de résultat à la charge de l'État. 
administratif de dégager une obligation de résultat, et donc un droit effectif à l'accès au service public de l'éducation. Les lois du service public n'épuisent pas toutes les potentialités des "droits à " dès lors que ces droits sont affirmés en des termes dénués d'équivoque ${ }^{1134}$; ces différente dispositions tendent au contraire à se combiner pour se renforcer mutuellement. En revanche, s'agissant $\mathrm{du}$ service public de la protection sociale, l'imprécision de l'alinéa $11 \mathrm{du}$ Préambule de la Constitution de 1946, qui en constitue le fondement textuel, n'autorise pas à espérer une solution analogue à celle retenue pour le service de l'éducation. Cela démontre ainsi l'importance de l'existence d'un texte de référence suffisamment précis dans l'activation de la contrainte pesant sur les personnes publiques, la consécration d'un droit d'accès effectif semble être subordonnée à cette condition.

Mais au-delà de ces considérations ayant trait à l'existence d'une disposition claire et précise, d'autres explications permettent de comprendre pourquoi le juge administratif se refuse généralement à reconnaître une obligation de résultat. Ces explications tiennent à la place que la jurisprudence administrative accorde à la liberté du commerce et de l'industrie, liberté qui peut se trouver remise en cause par la consécration d'un droit d'accès effectif au service public. Sur ce point, les décisions rendues à propos des arrêtés municipaux visant à interdire les coupures d'eau et d'énergie le confirment. Si le juge administratif n'exclut pas totalement que le maire fasse usage de son pouvoir de police en pareil cas, il estime cependant que ce pouvoir doit se concilier avec la liberté du commerce et de l'industrie ${ }^{1135}$. Or, les interdictions générales et absolues prises par les maires sont susceptibles de porter atteinte à cette liberté, et aucune justification tirée de la nécessité de préserver la dignité humaine n'a été admise par le juge ${ }^{1136}$. Même dans les hypothèses où l'arrêté était circonscrit aux seules personnes de bonne foi, le juge administratif a sanctionné l'illégalité en refusant de reconnaître qu'existait effectivement un risque pour la dignité humaine ${ }^{1137}$.

Dans le cadre des services publics industriels et commerciaux, le juge administratif a ainsi développé une jurisprudence restrictive à l'égard de l'effectivité du droit d'accès au service public, le principe de la liberté du commerce et de l'industrie expliquant sans doute que le juge répugne à mettre à la charge de l'État une obligation de résultat. Si ce principe cède parfois face à la nécessité de créer un service destiné à garantir à tous le bénéfice d'une prestation jugée indispensable ${ }^{1138}$, il s'oppose néanmoins, dans le cadre des services public industriels et commerciaux existants, à la mise en œuvre de dispositifs sociaux non prévus par les textes.

\footnotetext{
1134 Pour une analyse divergente, v. Marc PICHARD, Le droit à : Etude de législation française, Economica, collection recherches juridiques, 2006, p. 357 et s.

1135 V. en ce sens CAA de Versailles, 25 octobre 2007, commune de Bobigny, n06VE00008.

1136 Stéphane BRACONNIER, "Les arrêtés municipaux anti-coupures d'eau: une réponse juridique inadaptée à un problème social réel ", AJDA, 2005, p. 644.

1137 CAA Paris, 12 février 2008, Société EDF, nº 07PA02710.

1138 CE, 3 mars 2010, Département de la Corrèze, précit.
} 
En définitive, puisque le droit au service public est, pour reprendre le titre de cette contribution, le reflet des obligations pesant sur les personnes publiques, force est de constater que ce reflet est quelque peu trouble. Le droit à la création de services publics bénéficie en effet d'une opposabilité incertaine, dont le champ d'action paraît sectoriel. Quant à l'analyse de la créance pesant sur les personnes publiques à l'égard des services existants, elle met en lumière les limites de la justiciabilité d'un droit, en ce sens qu'elle n'est pas nécessairement garante de son effectivité. Mais dans tous les cas, l'existence de normes claires et précises permettrait sans doute d'accroître la contrainte pesant sur les personnes publiques.

\section{Pour citer cet article}

Virginie Donier, « Le droit au service public, reflet des obligations pesant sur les personnes publiques » La Revue des Droits de l'Homme, juin 2012

http://revdh.files.wordpress.com/2012/04/le-droit-au-service-public.pdf 